This could be related to hormonal changes [Padilha et al (1994) Reprod Nutr Dev (in press)].

\section{Metabolism and endocrinology}

\author{
Effect of insulin, and oleic acid on tri- \\ glyceride secretion by chicken hepato- \\ cytes in primary culture. Role of the $\Delta 9$ \\ desaturase. $\mathrm{P}$ Legrand, $\mathrm{E}$ Le Bihan, \\ D Catheline, MC Fichot, P Lemarchal (INRA, \\ Laboratoire de Biochimie, 65, rue de Saint- \\ Brieuc, 35000 Rennes, France)
}

Our aim is the study of the mechanisms which underlie fattening in the chicken. In this species, the liver is the main site for fatty-acid and triglyceride (TG) biosynthesis. The TG are secreted and transported in the blood in the form of very low density lipoprotein (VLDL) towards tissues for utilization or storage (adipose tissue). We developed a primary culture system of chicken hepatocytes in order to study the TG synthesis and secretion, by incorporation of $3 \mathrm{H}$-glycerol into intracellular and secreted TG. We first proposed a new method for secretion measurement, since we considered that simple medium removal underestimated TG secretion. Indeed, an important amount of VLDL seemed to be trapped in the unstirred water layer around the cells. Thus, after removing the medium, we incubated the cells briefly with trypsin to obtain a second fraction of medium and the cells. We first demonstrated that $90 \%$ of the labelled TG were in the form of VLDL in both medium fractions. We observed that the TG secretion increased at the beginning of the culture and reached a plateau after 1 or 2 $\mathrm{d}$ of culture. The secretion rate in the absence of insulin varied between 3 and $20 \mathrm{nmol} \mathrm{TG} / \mathrm{mg}$ cell protein/h depending on the donor animal. Among regulating factors, insulin has a controversial effect on TG secretion. We report here a clear significant activating effect of insulin. Labelled glycerol incorporation into secreted TG was 2.3-fold higher if insulin was present in the culture medium. Another activating factor is the availability of oleic acid (produced by the $\Delta 9$ desaturase). We proposed this hypothesis since we studied lipid metabolism in the fat and lean lines of chickens (selected by Leclercq, INRA, Tours) and reported that both hepatic $\Delta 9$ desaturase activity and plasma VLDL-TG concentration were higher in the fat animals than in the lean ones. In order to elucidate the role of oleic acid on TG secretion, we first investigated and identified a regulated $\Delta 9$ desaturase activity in cultured hepatocytes. We then blocked the $\Delta 9$ desaturase activity with a specific inhibitor (sterculic acid), and observed that TG secretion was significantly reduced whereas TG synthesis remained unchanged. Moreover, the addition of oleic acid in the culture medium, in the presence of the $\Delta 9$ desaturase inhibitor, partly restored the TG secretion. Our results demonstrate that oleic acid is a limiting factor for the TG secretion process, and that the $\Delta 9$ desaturase plays an important role in this process.

Influence of amino-acid concentration and insulin in culture media on aminoacid and glucose incorporation into cellular and secreted (VLDL) lipids by chicken hepatocytes in primary culture. JP Caffin $1,2\left({ }^{1}\right.$ Université Pierre-et-MarieCurie; 2 INRA, Station de Recherches Avicoles, 37380 Nouzilly, France)

The liver is the main site of lipogenesis in birds. Hence, the hepatocytes are a useful model for studying the mechanisms controlling lipogenesis.

Hepatocytes were isolated from broiler-type chicken (6-7 weeks old) by collagenase dispersion. The cells were plated at a density of $5.10^{5}$ cells $/ \mathrm{cm}^{2}$ at $37^{\circ} \mathrm{C}$ under a controlled atmosphere $\left(95 \%\right.$ air and $5 \% \mathrm{CO}_{2}$ ) in a medium containing Earle's balanced salts supplemented with MEMvitamins, glucose $(2 \mathrm{~g} / \mathrm{l})$, amino acids $(2.38 \mathrm{~g} / \mathrm{l})$, fetal calf serum (2.5\%), and chicken serum (3.5\%) in plastic 6-well plates. After plating, the medium was replaced with medium without fetal calf serum or chicken serum, with variable amino-acid concentrations $(0.3-5.96 \mathrm{~g} / \mathrm{l})$ and with or without insulin (100 nM). After the time of preincubation the medium was supplemented with ${ }^{3} \mathrm{H}$-glycerol $(25 \mathrm{nM})$ and $\mathrm{U}^{14} \mathrm{C}$-protein hydrolysate or $\mathrm{U}^{14} \mathrm{C}$ glucose and the incubation was continued for 2-24 h. The incorporation was stopped by medium aspiration and the cells were lysed by freeze-thawing in water. The chloroform/methanol extracts of medium and cells were used for radioactivity determination.

The increase in amino-acid concentration in culture media increased incorporation of amino acids and glucose into cellular and secreted lipids (VLDL) with a cooperative effect on glucose incorporation into secreted lipids (VLDL). Increases 
in amino-acid concentration enhanced aminoacid and glucose utilization for lipogenesis, with no increase of lipogenesis measured by glycerol incorporation for incubation times below $10 \mathrm{~h}$.

Insulin increased glycerol incorporation ( $+35 \%)$ into cellular lipids and consequently lipogenesis. Insulin increased more strongly amino-acid and glucose incorporation into cellular lipids, the glucose incorporation showed a synergistic effect between insulin and amino acids for up to $1.59 \mathrm{~g} / \mathrm{l}$ amino acid in the medium (increase in the presence of insulin was of 2 at $0.3 \mathrm{~g} / \mathrm{l}$ to 10.6 at 1.59 $\mathrm{g} / \mathrm{l})$. At concentrations above $1.59 \mathrm{~g} / \mathrm{l}$, the synergistic effect decreased (5-6); this is in accordance with the inhibitory effect of amino acids on insulin stimulation of amino-acid transport by hepatocytes in primary culture.

In contrast, insulin decreased lipid secretion measured by glycerol $(-35 \%)$ and glucose incorporation. This decreased glycerol incorporation was correlated with the medium amino-acid concentration. When the amino-acid concentration was lower than $1.19 \mathrm{~g} / \mathrm{l}$, insulin increased aminoacid incorporation, but it decreased amino-acid incorporation if the concentration exceeded this value. This effect accounts for the controversy over the insulin action on VLDL secretion.

\section{Substrate utilization by bovine and ovine adipose tissue explants cultured for 2-7 d: effects of insulin and/or dexame-}

thasone. Y Faulconnier, L Guillon, J Fléchet, Y Chilliard (INRA, Laboratoire SousNutrition des Ruminants, 63122 SaintGenès-Champanelle, France)

Effects of crystalline bovine insulin (Sigma, 2 $\mathrm{mU} / \mathrm{mL}$ ) and/or dexamethasone (Dex, Sigma, $100 \mathrm{nM}$ ) were studied on perirenal adipose tissue (AT) from non-lactating non-pregnant cows ( $n$ $=5)$ and ewes $(n=5)$ fed on a restricted diet which provided $20-22 \%$ of the energy maintenance requirement, EMR for 8-10 $d$ and then overfed ( $188 \%$ or $228 \%$ of the EMR, for cows or ewes) for 21 (cows) or 10 (ewes) d until slaughter. AT explants were cultured in sterile conditions for $2-7 \mathrm{~d}$. The amount of glucose removed from the culture medium was determined daily for $7 \mathrm{~d}$ using the glucose dehydrogenase method and acetate utilization for $\mathrm{d} 1-2,3-4$ and 5-6-7 using an enzymatic method. Culture medium contained $5.5 \mathrm{mM}$ glucose and $7.6 \mathrm{mM}$ acetate, and was changed daily. In basal conditions, glucose and acetate utilization was similar in bovine and ovine AT explants (table I). Acetate utilization on d 1-2 was 3 times greater than glucose utilization, but they were similar after $d 4$.

The addition of insulin tended to increase glucose utilization by bovine AT explants. However, the effect of insulin on $d 3-4$ and $d 5-7$ was greater in ovine $(112 \%$ increase) than in bovine AT $(+57 \%)$. The addition of Dex to the insulinsupplemented medium increased further $(P<$

Table I. Effects of insulin $(\mathrm{I}, 2 \mathrm{mU} / \mathrm{ml})$ and/or dexamethasone $(\mathrm{D}, 100 \mathrm{nM})$ on glucose and acetate utilization by bovine and ovine adipose tissue in culture ( $Y$ Faulconnier et al).

\begin{tabular}{|c|c|c|c|c|c|c|c|c|}
\hline & \multicolumn{4}{|c|}{ Days 1-2 } & \multicolumn{4}{|c|}{ Days 5-7 } \\
\hline & Control & 1 & $D$ & $I D$ & Control & 1 & $D$ & $I D$ \\
\hline \multicolumn{9}{|l|}{ Glucose 1} \\
\hline Bovine & $31^{\mathrm{ab}}$ & $49^{a}$ & $17^{b}$ & $39 a$ & 17 & 26 & 18 & 19 \\
\hline Ovine & $36^{b}$ & $51^{a}$ & $16^{c}$ & $50^{a}$ & $15^{\mathrm{c}}$ & $35^{b}$ & $9^{c}$ & $52^{a}$ \\
\hline \multicolumn{9}{|l|}{ Acetate 1} \\
\hline Bovine & $95^{b}$ & $117^{a b}$ & $47^{c}$ & $132^{\mathrm{a}}$ & $20^{b}$ & $55^{a}$ & $13^{b}$ & $70^{a}$ \\
\hline Ovine & $99^{a}$ & $103^{a}$ & $49 b$ & $114^{a}$ & $12^{\mathrm{bc}}$ & $29^{b}$ & $6^{c}$ & $65^{a}$ \\
\hline
\end{tabular}

\footnotetext{
1 Utilisation as $\mu \mathrm{mol} / 24 \mathrm{~h} / 10^{6}$ adipocytes. a,b,c means in the same row and for the same culture duration with different letters differ $(P<0.05)$. The adipocyte number of explants was calculated from the mean cell volume determined after tissue fixation in osmium tetroxide.
} 